\title{
AUXILIARY FUNCTIONS IN THE STUDY OF STEFAN-LIKE PROBLEMS WITH VARIABLE THERMAL PROPERTIES
}

\author{
ANDREA N. CERETANI *, NATALIA N. SALVA ${ }^{\dagger}$, AND DOMINGO A. TARZIA $\ddagger$
}

\begin{abstract}
We address the existence and uniqueness of the so-called modified error function that arises in the study of phase-change problems with specific heat and thermal conductivity given by linear functions of the material temperature. This function is defined from a differential problem that depends on two parameters which are closely related with the slopes of the specific heat and the thermal conductivity. We identify conditions on these parameters which allow us to prove the existence of the modified error function. In addition, we show its uniqueness in the space of nonnegative bounded analytic functions for parameters that can be negative and different from each other. This extends known results from the literature and enlarges the class of associated phasechange problems for which exact similarity solutions can be obtained. In addition, we provide some properties of the modified error function considered here.
\end{abstract}

Key words. Modified error function, Stefan problems, temperature-dependent thermal coefficients, nonlinear boundary value problems

AMS subject classifications. 34B15, 34B08, 80A22, 35R35

1. Introduction. This article is devoted to show the existence and uniqueness of an auxiliary function that arise in the study of phase-change processes when some thermal coefficients are assumed to vary with the material temperature. The function of interest is the solution to the following nonlinear differential system:

$$
\left((1+\delta y) y^{\prime}\right)^{\prime}+2 x(1+\gamma y) y^{\prime}=0 \quad x \in(0,+\infty), \quad y(0)=0, \quad y(+\infty)=1,
$$

where $\delta, \gamma \in(-1,+\infty)$, and $y(+\infty):=\lim _{x \rightarrow+\infty} y(x)$.

Problem (1.1) was introduced by Oliver and Sunderland in 11 for the study of two-phase Stefan problems on the semi-infinite line $(0,+\infty)$, with heat capacity and thermal conductivity given by

$$
c(\vartheta):=c_{\mathrm{o}}\left(1+\alpha \frac{\vartheta-\vartheta_{\mathrm{o}}}{\vartheta_{\mathrm{i}}-\vartheta_{\mathrm{o}}}\right) \quad \text { and } \quad k(\vartheta):=k_{\mathrm{o}}\left(1+\beta \frac{\vartheta-\vartheta_{\mathrm{o}}}{\vartheta_{\mathrm{i}}-\vartheta_{\mathrm{o}}}\right),
$$

respectively, where $\vartheta$ is the material temperature, $c_{\mathrm{o}}>0$ and $k_{\mathrm{o}}>0$ are reference values for the specific heat and the thermal conductivity, $\vartheta_{\mathrm{i}}$ is a uniform initial temperature distribution, $\vartheta_{\mathrm{o}}$ is a prescribed constant temperature at the boundary $x=0$, and $\alpha, \beta \in \mathbb{R}$ (the values of $\alpha, \beta, c_{\mathrm{o}}$, and $k_{\mathrm{o}}$ generally differ from liquid to solid phases). In particular, they proposed a method to find similarity solutions that relies on the assumption that problem (1.1) has a solution $\Phi_{\delta \gamma}$ for any $\delta, \gamma \in(-1,+\infty)$. The temperature in each phase is then obtained in terms of an auxiliary function $\Phi_{\delta \gamma}$ for some parameters $\delta$ and $\gamma$ that must be calculated and may be different from one phase to

*IMAS-UBA-CONICET, Intendente Guiraldes 2160, Capital Federal 1428, Argentina, aceretani@dm.uba.ar, and Escuela de Ciencia y Tecnología, Universidad Nacional de San Martín, Martín de Irigoyen 3100, San Martín 1650, Argentina, aceretani@unsam.edu.ar

${ }^{\dagger}$ CNEA-CONICET, Departamento de Mecánica Computacional, Centro Atómico Bariloche, Av. Bustillo 9500, Bariloche 8400, Argentina, and Departamento de Matemática, Centro Regional Bariloche, Universidad Nacional del Comahue, Quintral 1250, Bariloche 8400, Argentina, natalia@cab.cnea.gov.ar

${ }^{\ddagger}$ CONICET-Departamento de Matemática, Facultad de Ciencias Empresariales, Universidad Austral, Paraguay 1950, Rosario 2000, Argentina, dtarzia@austral.edu.ar 
the other, see equations (23)-(27) in [1]. The function $\Phi_{\delta \gamma}$ was called modified error function and it was obtained numerically by solving problem (1.1).

The signs of $\delta$ and $\gamma$ are closely related on how the thermal conductivity and the heat capacity vary with the material temperature. We illustrate this in the following example: Consider a one-phase solidification process for a material with phase-change temperature $\vartheta_{\mathrm{f}}$. Then, $\vartheta_{\mathrm{o}}<\vartheta_{\mathrm{f}}$ and $\vartheta_{\mathrm{i}} \equiv \vartheta_{\mathrm{f}}$. According with [1, it must be $\gamma \Phi_{\delta \gamma}(\lambda)=$ $\alpha$ and $\delta \Phi_{\delta \gamma}(\lambda)=\beta$, where $\lambda \equiv \frac{s(t)}{2 \sqrt{a t}}$ for all $t>0, x=s(t)$ is the location of the free boundary at time $t$, and $a$ is the coefficient of diffusion of the solid phase. Then, provided that $\alpha \neq 0$ and $\beta \neq 0$ we have $\operatorname{sign}(\gamma)=\operatorname{sign}(\alpha)$ and $\operatorname{sign}(\delta)=\operatorname{sign}(\beta)$ since the modified error function is non-negative everywhere (see Theorem 2.1 below). We now observe that the slopes of $c$ and $k$ are given by $\frac{c_{\circ} \alpha}{\vartheta_{\mathrm{i}}-\vartheta_{\mathrm{o}}}$ and $\frac{k_{\mathrm{o}} \beta}{\vartheta_{\mathrm{i}}-\vartheta_{\mathrm{o}}}$, respectively, see (1.2), where $\vartheta_{\mathrm{i}}-\vartheta_{\mathrm{o}}>0$. Therefore, the heat capacity is increasing for $\gamma>0$, decreasing for $\gamma<0$, and the analogous conclusion holds for the relation between the thermal conductivity and the parameter $\delta$.

The method described in [1] had already been considered by Cho and Sunderland in 2 for the analogous Stefan problem with constant heat capacity, corresponding to $\gamma=0$ in (1.1). The modified error function for the case when $\gamma=0$ and $\delta>0$ was studied by the authors in [3] (see also [5]), where existence and uniqueness in the space of bounded analytic functions were proven and explicit approximations were provided (see [6] for improved approximations). In particular, this paper extends the existence and uniqueness result in [3] for the case $\delta<0(\gamma=0)$.

Similar approaches to those introduced by Sunderland and collaborators were followed to find exact similarity solutions in the cases when non-Dirichlet boundary conditions are prescribed at $x=0$ or when the physical domain is allowed to move, see e.g. [7 9]. In all cases it was assumed that $\alpha=\beta>0$, or $\alpha=0$ and $\beta>0$. Analogous methods were also used to determine solutions to problems with more general thermal coefficients, see e.g. [10,11. Other approaches to find similarity solutions to Stefanlike problems with non-constant thermal properties and arbitrary initial and boundary conditions were recently considered in, e.g., 12 16. We refer to [1 for a discussion about the effects of including variations with respect to the material temperature of thermal conductivity and heat capacity in phase-change models.

In the next section we provide the existence and uniqueness of a solution $\Phi_{\delta \gamma}$ to problem (1.1) in the space of bounded analytic functions, for some $\delta, \gamma \in(-1,+\infty)$. In particular, $\delta$ and $\gamma$ are allowed to be negative and different from each other. In this manner we extend already known results for modified error functions available in the literature.

2. Existence and uniqueness of $\Phi_{\delta \gamma}$. The following is the main result of the paper.

Theorem 2.1. Assume that

$M(\delta, \gamma):=\frac{\max (1,1+\delta)^{3 / 2} \max (1,1+\gamma)^{1 / 2}}{\min (1,1+\delta)^{5 / 2} \min (1,1+\gamma)^{1 / 2}}\left(2|\delta|+\frac{|\delta-\gamma| \max (1,1+\delta)}{\min (1,1+\delta) \min (1,1+\gamma)}\right)<1$.

Then problem (1.1) admits a unique bounded analytic solution $\Phi_{\delta \gamma}$ that satisfies

$$
0 \leq \Phi_{\delta \gamma}(x) \leq 1 \quad \text { for all } \quad x \geq 0
$$


Proof. Let $X$ be the Banach space of bounded analytic functions $h:[0,+\infty) \rightarrow \mathbb{R}$, equipped with the supremum norm $\|h\|_{\infty}:=\sup \{|h(x)|: x \geq 0\}$. In addition, let

$$
K:=\left\{h \in X:\|h\|_{\infty} \leq 1, h \geq 0, h(0)=0\right\} .
$$

We notice that $K$ is a non-empty closed subset of $X$.

Let $h \in K$. Consider the following auxiliary linear problem:

$$
\left((1+\delta h) y^{\prime}\right)^{\prime}+2 x(1+\gamma h) y^{\prime}=0 \quad x \in(0,+\infty), \quad y(0)=0, \quad y(+\infty)=1 .
$$

From this, we shall formulate the existence and uniqueness of a solution to (1.1) as a fixed point problem.

Let $F(\cdot ; h):[0,+\infty) \rightarrow \mathbb{R}$ be given by

$$
F(x ; h):=\int_{0}^{x} \exp \left(-\int_{0}^{w} \frac{2 z(1+\gamma h(z))}{1+\delta h(z)} \mathrm{d} z\right) \frac{1}{1+\delta h(w)} \mathrm{d} w .
$$

Exploiting the inequalities

$$
0<\min (1,1+v) \leq 1+v h \leq \max (1,1+v), \quad \text { for } \quad v=\delta \quad \text { or } \quad v=\gamma,
$$

and taking into account that $\int_{0}^{+\infty} \exp \left(-w^{2}\right) \mathrm{d} w=\frac{\sqrt{\pi}}{2}$, we obtain

$$
0 \leq F(x ; h) \leq \frac{\sqrt{\pi} \max (1,1+\delta)^{1 / 2}}{2 \min (1,1+\delta) \min (1,1+\gamma)^{1 / 2}}=: M_{1}(\delta, \gamma) \quad \text { for all } \quad x \geq 0 .
$$

Then $F(\cdot ; h)$ is well-defined. Analogous computations allow us to observe

$$
\frac{1}{F(+\infty ; h)} \leq \frac{2 \max (1,1+\delta) \max (1,1+\gamma)^{1 / 2}}{\sqrt{\pi} \min (1,1+\delta)^{1 / 2}}=: M_{2}(\delta, \gamma)
$$

Hence, the map $T: K \rightarrow K$ given by

$$
(T(h))(x):=\frac{F(x ; h)}{F(+\infty ; h)},
$$

is well-defined too. Then, we notice that a necessary and sufficient condition to be $y \in K$ a solution to (1.1) is that $T(y)=y$ since $y=T(h)$ solves (2.2) for each $h \in K$. The rest of the proof consists of proving that $T$ is a contraction from $K$ into itself, provided (2.1) holds true. Then, the theorem will follow by Banach's fixed point theorem.

Initially, we note $T(K) \subset K$ as a direct consequence of the definition of $T$. Let $x \geq 0$ and $h_{1}, h_{2} \in K$. We have:

$$
\begin{aligned}
\left|\left(T\left(h_{1}\right)\right)(x)-\left(T\left(h_{2}\right)\right)(x)\right| & \leq\left|\frac{F\left(x ; h_{1}\right)}{F\left(+\infty ; h_{1}\right)}-\frac{F\left(x ; h_{2}\right)}{F\left(+\infty ; h_{1}\right)}\right|+\left|\frac{F\left(x ; h_{2}\right)}{F\left(+\infty ; h_{1}\right)}-\frac{F\left(x ; h_{2}\right)}{F\left(+\infty ; h_{2}\right)}\right| \\
& \leq \frac{\left|F\left(x ; h_{1}\right)-F\left(x ; h_{2}\right)\right|}{F\left(+\infty ; h_{1}\right)}+\frac{\left|F\left(+\infty ; h_{2}\right)-F\left(+\infty ; h_{1}\right)\right|}{F\left(+\infty ; h_{1}\right)} .
\end{aligned}
$$

Then, using (2.4) we find

$$
\left|\left(T\left(h_{1}\right)\right)(x)-\left(T\left(h_{2}\right)\right)(x)\right| \leq M_{2}(\delta, \gamma)(I+J),
$$


where $I:=\left|F\left(x ; h_{1}\right)-F\left(x ; h_{2}\right)\right|$ and $J:=\left|F\left(+\infty ; h_{1}\right)-F\left(+\infty ; h_{2}\right)\right|$.

Defining $f(w):=\exp (-2 w)$ and $w_{i}:=\int_{0}^{w} \frac{z\left(1+\gamma h_{i}(z)\right)}{1+\delta h_{i}(z)} \mathrm{d} z$ for $w \geq 0, i=1,2$, and using the estimates (2.3), we have

$$
\begin{aligned}
I & \leq\left|\int_{0}^{x} \frac{f\left(w_{1}\right)}{1+\delta h_{1}(w)}-\frac{f\left(w_{2}\right)}{1+\delta h_{1}(w)} \mathrm{d} w\right|+\left|\int_{0}^{x} \frac{f\left(w_{2}\right)}{1+\delta h_{1}(w)}-\frac{f\left(w_{2}\right)}{1+\delta h_{2}(w)} \mathrm{d} w\right| \\
& \leq \frac{1}{\min (1,1+\delta)} \int_{0}^{x}\left|f\left(w_{1}\right)-f\left(w_{2}\right)\right| \mathrm{d} w+\frac{|\delta|\left\|h_{1}-h_{2}\right\|_{\infty}}{\min (1,1+\delta)^{2}} \int_{0}^{x} f\left(w_{2}\right) \mathrm{d} w .
\end{aligned}
$$

Let $w \geq 0$. From the Mean Value Theorem we observe that $f\left(w_{1}\right)-f\left(w_{2}\right)=$ $-2 f(\omega)\left(w_{1}-w_{2}\right)$, where $\omega$ is a number in between $w_{1}$ and $w_{2}$. Furthermore, since $f$ is decreasing we have that $f(\omega) \leq f\left(\min \left(w_{1}, w_{2}\right)\right)=f\left(w_{k}\right)$ for $k=1$ or $k=2$. Then,

$$
\begin{aligned}
\int_{0}^{x}\left|f\left(w_{1}\right)-f\left(w_{2}\right)\right| \mathrm{d} w & \leq 2 \int_{0}^{x} f\left(w_{k}\right)\left|w_{1}-w_{2}\right| \mathrm{d} w \\
& \leq \frac{|\delta-\gamma|\left\|h_{1}-h_{2}\right\|_{\infty}}{\min (1,1+\delta)^{2}} \int_{0}^{x} w^{2} \exp \left(-\frac{\min (1,1+\gamma)}{\max (1,1+\delta)} w^{2}\right) \mathrm{d} w \\
& \leq \frac{\sqrt{\pi}|\delta-\gamma| \max (1,1+\delta)^{3 / 2}}{4 \min (1,1+\delta)^{2} \min (1,1+\gamma)^{3 / 2}}\left\|h_{1}-h_{2}\right\|_{\infty}
\end{aligned}
$$

where we have used (2.3) and that $\int_{0}^{+\infty} w^{2} \exp \left(-w^{2}\right) \mathrm{d} w=\frac{\sqrt{\pi}}{4}$.

From analogous arguments, we find

$$
\int_{0}^{x} f\left(w_{2}\right) \mathrm{d} w \leq \frac{\sqrt{\pi} \max (1,1+\delta)^{1 / 2}}{2 \min (1,1+\gamma)^{1 / 2}} .
$$

Hence, using (2.8) and (2.9) on (2.7), we obtain $I \leq M_{3}(\delta)\left\|h_{1}-h_{2}\right\|_{\infty}$, where

$$
M_{3}(\delta):=\frac{\sqrt{\pi} \max (1,1+\delta)^{1 / 2}}{4 \min (1,1+\delta)^{2} \min (1,1+\gamma)^{1 / 2}}\left(2|\delta|+\frac{|\delta-\gamma| \max (1,1+\delta)}{\min (1,1+\delta) \min (1,1+\gamma)}\right) .
$$

An identical argument yields $J \leq M_{3}(\delta, \gamma)\left\|h_{1}-h_{2}\right\|_{\infty}$. Hence, it follows from (2.6) and the above estimates for $I$ and $J$ that

$$
\left\|T\left(h_{1}\right)-T\left(h_{2}\right)\right\|_{\infty} \leq 2 M_{2}(\delta, \gamma) M_{3}(\delta, \gamma)\left\|h_{1}-h_{2}\right\|_{\infty}=M(\delta, \gamma)\left\|h_{1}-h_{2}\right\|_{\infty},
$$

Therefore, condition (2.1) ensures that $T$ is a contracting map and the proof is finished.

REMARK 1. Theorem [2.1 improves the analogous result given by the authors in [3] for the case when $\delta>0$ and $\gamma=0$. In fact, the result given in [3] holds true provided that $\delta>0$ satisfies $\frac{\delta}{2}(1+\delta)^{3 / 2}(3+\delta)\left(1+(1+\delta)^{3 / 2}\right)<1$, whereas condition (2.1) in Theorem 2.1 just requires $\delta(1+\delta)^{3 / 2}(3+\delta)<1$.

Figure 2.1a depicts the set $\mathcal{A}$ of all ordered pairs $(\delta, \gamma)$ that satisfy condition (2.1) in Theorem 2.1. Figures 2.1b and 2.1c show plots of the modified error function $\Phi_{\delta \gamma}$ for several choices of the parameters $\delta$ and $\gamma$, obtained by solving numerically the boundary value problem (1.1) through the bvodes routine implemented in Scilab [17. 


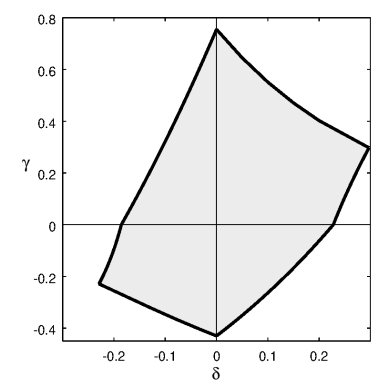

(a) $\mathcal{A}=\{(\delta, \gamma): M(\delta, \gamma)<1\}$

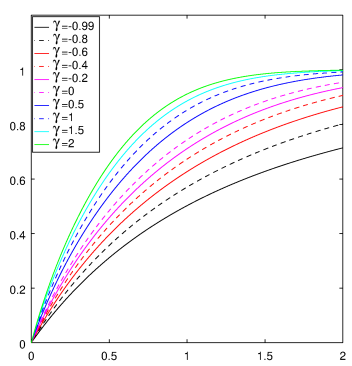

(b) $\Phi_{\delta \gamma}$ for $\delta=1.5$

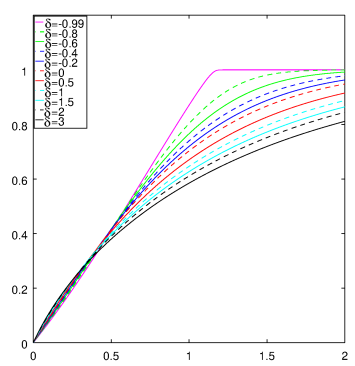

(c) $\Phi_{\delta \gamma}$ for $\gamma=-0.6$

Fig. 2.1: Pairs $(\delta, \gamma)$ that satisfy condition (2.1) in Theorem 2.1 (left) and plots of the modified error function $\Phi_{\delta \gamma}$ for $\delta=1.5$ (middle) and $\gamma=-0.6$ (right).

Note that condition (2.1) is sufficient but not necessary, therefore we show the plots of the modified error function for parameters included and not included in the set $\mathcal{A}$.

The next corollary closes the article and establishes that the modified error function $\Phi_{\delta \gamma}$ given by Theorem 2.1 shares some essential features with the classical error function. The proof is analogous to the one for Theorem 5.1 of [4] so that we shall omit it here.

Corollary 2.2. Suppose that (2.1) holds true. Then the unique solution $\Phi_{\delta \gamma}$ to problem (1.1) given by Theorem 2.1 is increasing. If in addition $\delta$ is non-negative, then $\Phi_{\delta \gamma}$ is also concave.

Acknowledgments. D. A. Tarzia and N. N. Salva have been supported by CONICET within the project PIP 0275, Universidad Austral, Rosario, Argentina and by the European Union's Horizon 2020 Research and Innovation Programme within the Marie Sklodowska-Curie grant agreement 823731 CONMECH.

\section{REFERENCES}

[1] D. L. R. Oliver and J. E. Sunderland. A phase-change problem with temperature-dependent thermal conductivity and specific heat. Int. J. Heat Mass Transfer, 30:2657-2661, 1987.

[2] S. H. Cho and J. E. Sunderland. Phase-change problems with temperature-dependent thermal conductivity. Journal of Heat Transfer, 96(2):214-217, 1974.

[3] A. N. Ceretani, N. N. Salva, and D. A. Tarzia. Existence and uniqueness of the modified error function. Applied Mathematics Letters, 70:14-17, 2017.

[4] A. N. Ceretani, N. N. Salva, and D. A. Tarzia. Approximation of the modified error function. Applied Mathematics and Computations, 337:607-617, 2018.

[5] L. Bougoffa. A note on the existence and uniqueness solutions of the modified error function. Mathematical Methods in the Applied Sciences, 41(14):5526-5534, 2018.

[6] S. Mandal, D. Singh, and M. M. Panja. A note on corrections in approximation of the modified error function. Journal of Advances in Mathematics and Computer Science, 30(5):1-13, 2019.

[7] A. N. Ceretani, N. N. Salva, and D. A. Tarzia. An exact solution to a Stefan problem with variable thermal conductivity and a robin boundary condition. Nonlinear Analysis: Real World Applications, 40:243-259, 2018.

[8] A. Kumar Singh, A. Kumar, and Rajeev. Exact and approximate solutions of a phase change problem with moving phase change material and variable thermal coefficients. Journal of King Saud University - Science, 2018, in press.

[9] A. Kumar Singh, A. Kumar, and Rajeev. A Stefan problem with variable thermal coefficients 
and moving phase change material. Journal of King Saud University - Science, 2018, in press.

[10] J. Bollati, M. F. Natale, J. A. Semitiel, and D. A. Tarzia. Existence and uniqueness of solution for two one-phase Stefan problems with variable thermal coefficients. Nonlinear Analysis: Real World Applications, 51:103001, 2020.

[11] A. Kumar, A. Kumar Singh, and Rajeev. A moving boundary problem with variable specific heat and thermal conductivity. Journal of King Saud University - Science, 2018, in Press.

[12] A. C. Briozzo and M. F. Natale. Non-classical Stefan problem with nonlinear thermal coefficients and a Robin boundary condition. Nonlinear Analysis: Real World Applications, 49:159-168, 2019.

[13] F. Font. A one-phase Stefan problem with size-dependent thermal conductivity. Applied Mathematical Modelling, 63:172-178, 2018.

[14] Y. Zhou, Y.J. Wang, and W.K. Bu. Exact solution for a Stefan problem with latent heat a power function of position. Int. J. of Heat and Mass Transfer, 69:451-454, 2014.

[15] J. Bollati and D. A. Tarzia. One-phase Stefan problem with a latent heat depending on the position of the free boundary and its rate of change. Electronic Journal of Differential Equations, 2018(10):1-12, 2018.

[16] L.N. Tao. The Stefan problem with arbitrary initial and boundary conditions. Quart. Appl. Math., 36:223-233, 1978.

[17] U. Ascher, J. Christiansen, and R.D. Russell. Collocation software for boundary-value odes. ACM Trans. on Math. Software, 7:209-222, 1981. 